\title{
Identification of protein complexes and functional modules in E. coli PPI networks
}

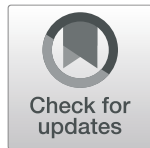

Ping Kong ${ }^{1}$, Gang Huang ${ }^{1}$ and Wei Liu ${ }^{2,3^{*}}$ (D)

\begin{abstract}
Background: Escherichia coli always plays an important role in microbial research, and it has been a benchmark model for the study of molecular mechanisms of microorganisms. Molecular complexes, operons, and functional modules are valuable molecular functional domains of $E$. coli. The identification of protein complexes and functional modules of $E$. coli is essential to reveal the principles of cell organization, process, and function. At present, many studies focus on the detection of $E$. coli protein complexes based on experimental methods. However, based on the large-scale proteomics data set of $E$. coli, the simultaneous prediction of protein complexes and functional modules, especially the comparative analysis of them is relatively less.

Results: In this study, the Edge Label Propagate Algorithm (ELPA) of the complex biological network was used to predict the protein complexes and functional modules of two high-quality PPI networks of E. coli, respectively. According to the gold standard protein complexes and function annotations provided by EcoCyc dataset, most protein modules predicted in the two datasets matched highly with real protein complexes, cellular processes, and biological functions. Some novel and significant protein complexes and functional modules were revealed based on ELPA. Moreover, through a comparative analysis of predicted complexes with corresponding functional modules, we found the protein complexes were significantly overlapped with corresponding functional modules, and almost all predicted protein complexes were completely covered by one or more functional modules. Finally, on the same PPI network of E. coli, ELPA was compared with a well-known protein module detection method (MCL) and we found that the performance of ELPA and MCL is comparable in predicting protein complexes.

Conclusions: In this paper, a link clustering method was used to predict protein complexes and functional modules in PPI networks of E. coli, and the correlation between them was compared, which could help us to understand the molecular functional units of $E$. coli better.
\end{abstract}

Keywords: Protein complexes, Functional modules, Link clustering algorithm, E. coli PPI networks

\section{Background}

Escherichia coli (E. coli) is the primary model organism of microorganisms, and perhaps it is the most intensively studied species of bacteria [1-4]. Even so, only twothirds of the protein-coding gene products of E. coli K-

\footnotetext{
* Correspondence: liuyiwei1030@hotmail.com

${ }^{2}$ Research Center for Intelligence Information Technology, Nantong University, Nantong 226019, Jiangsu, China

${ }^{3}$ School of Mathematics and Statistics Science, Lu Dong University, Yantai 264025, Shandong, China

Full list of author information is available at the end of the article
}

12 currently have experimental evidence for their biological roles, and others remain unannotated (orphans) [5]. Experiments and data analysis (algorithmic model) are two effective methods to identify protein complexes and functional modules of E. coli. However, it is wellknown that experimental analysis has always been dominant because of the lack of large-scale experimental data and the incompleteness of the E. coli dataset [5-9]. Experimental methods have the advantages of direct verification, but they also have the limitations of high false positive rates and false negative rates. In recent years,

C C The Author(s). 2020 Open Access This article is licensed under a Creative Commons Attribution 4.0 International License, which permits use, sharing, adaptation, distribution and reproduction in any medium or format, as long as you give appropriate credit to the original author(s) and the source, provide a link to the Creative Commons licence, and indicate if changes were made. The images or other third party material in this article are included in the article's Creative Commons licence, unless indicated otherwise in a credit line to the material. If material is not included in the article's Creative Commons licence and your intended use is not permitted by statutory regulation or exceeds the permitted use, you will need to obtain permission directly from the copyright holder. To view a copy of this licence, visit http://creativecommons.org/licenses/by/4.0/ The Creative Commons Public Domain Dedication waiver (http://creativecommons.org/publicdomain/zero/1.0/) applies to the data made available in this article, unless otherwise stated in a credit line to the data. 
with the development of genomic technology, some high-throughput, high-quality, binary protein interaction (PPIs) maps of $E$. coli have been released, so the protein complexes and functional modules of $E$. coli and their relationships can be predicted from a global perspective [8-19]. Although the analysis methods of these data were not perfect, many studies showed that the prediction results of these methods are useful supplements to the experimental methods.

A protein complex is formed by the interaction of more than two functional related peptide chains through disulfide bonds or other proteins, so it performs some given biological functions. A functional module is the basic functional unit of proteins, which implies complex relationships involving multiple biological interaction types [8]. Revealing protein complexes and functional modules in the $E$. coli PPI network is an important research topic to understand the essential biological functions of proteins. Although some studies used complex network models to make predictions, due to the lack of large-scale PPI datasets and the existence of a large number of orphan proteins, the prediction results were difficult to achieve the expected ones. However, with the release of some high-throughput $E$. coli PPI datasets in recent years, it has become possible to predict protein complexes and functional modules of $E$. coli based on complex network models. With the development of high-throughput sequencing technologies, such as twohybrid systems and mass spectrometry technology for pairwise protein interactions, large-scale PPI networks of E. coli can be constructed at genome level [20]. Some studies explored the prediction of protein complexes based on E. coli PPI networks [5, 10, 11], and others focused on the functional relationship between transcription regulation [12-16] and metabolic pathway [17-19] of $E$. coli. Even so, studies of predicting protein complexes and functional modules at the same time, especially the comparative analysis of them is relatively less. In this paper, based on the $E$. coli datasets of $\mathrm{Hu}$ et al. [5] and Cong et al. [8], two complex-related PPI networks were constructed, named netH and netC, respectively. We focused on the recognition and analysis of protein complexes and functional modules in the two large-scale PPI networks, and discussed the differences and connections between them.

Node clustering and link clustering are two different methods to reveal the network structure from different perspectives. Because the link itself contains node attributes, link clustering has a natural advantage over the node clustering algorithm in the identification of network modules. In this study, E. coli protein complexes and functional modules were predicted by a link clustering method (ELPA [21]) in two high-quality PPI networks. Many studies showed that Markov Clustering algorithm (MCL) was an excellent protein module identification algorithm and the most popular method for detecting protein complexes [22-25], so we compared the results of ELPA with MCL on the same PPI network of E. coli. According to the gold-standard protein complexes and function annotations provided by EcoCyc dataset, the results showed that most protein modules predicted by ELPA matched well with real protein complexes, cellular processes, or biological functions, and the performance was comparable with MCL. For example, in the PPI network of $E$. coli provided by $\mathrm{Hu}$ et al., $75.8 \%$ of predicted protein modules matched with one or more real protein complexes, $88.1 \%$ of real protein complexes matched with one or more protein modules, and $88.3 \%$ of predicted protein modules matched with at least one functional unit of $E$. coli. Furthermore, some novel protein complexes and functional modules were uncovered in both networks, and we compared the protein complex with the corresponding functional module predicted from the same protein module. The results showed that the protein complex significantly overlapped with the corresponding functional module, and many functional modules contained more than one protein complex. Therefore, we concluded that ELPA is an effective method to predict protein complexes and functional modules in PPI networks of $E$. coli.

\section{Results}

\section{Identification of protein modules}

ELPA predicted 120 and 171 protein modules in netH and netC (Table S1), respectively. The size of predicted modules ranged from two to hundreds of proteins. Also, we found that many protein modules detected by ELPA overlapped each other. This phenomenon was very consistent with real protein complexes and functional modules, which meant that some proteins involved multiple complexes or functional modules. It is also an important research topic to study the overlapping proteins in different complexes or functional modules.

\section{Prediction of protein complexes}

Those protein modules of net $\mathrm{H}$ and netC identified by ELPA were matched with 295 real benchmark protein complexes of E. coli in the EcoCyc dataset, respectively. In netH, 222 benchmark complexes (75.3\%) matched 91 predicted protein modules (75.8\%), and in netC, 200 benchmark complexes $(67.8 \%)$ matched 144 predicted protein modules $(70.9 \%)$. Since most benchmark complexes consist of no more than ten proteins, larger protein modules may contain multiple complexes, which is consistent with the composition of real complexes.

When the Matching Score threshold was set to 0.2 [24, 26], 84 and 136 protein complexes were predicted in netH and netC, respectively. By comparing with the 
benchmark complexes, we found that most of the protein complexes predicted by ELPA matched well with the corresponding real complexes in both networks. For example, in netH, module 50 consisted of eight proteins, of which $p o t F$, potH, and potI were the three proteins in the putrescine $\mathrm{ABC}$ transporter complex; pot $A$, pot $B$, pot $C$, and pot $D$ covered all four proteins of the putrescine/spermidine $\mathrm{ABC}$ transporter complex (as shown in Fig. 1a). Module 54 protein module consisted of five proteins, which were completely covered by two complexes: the ferrichrome transport system and the ferric coprogen transport system. The ferrichrome transport system protein complex consisted of four proteins: fhuA, $f h u B$, fhuC, and $f h u D$, while the ferric coprogen transport system protein complex was composed of $f h u B$, fhuC, fhuD, and fhuE. As shown in Fig. 1b, it could be found that $f h u B, f h u C$, and $f h u D$ were the three proteins shared by these two complexes. In netC, module 37 consisted of four proteins, of which $u g p A, u g p B, u g p C$, and $u g p E$ were exactly the four proteins that made up the glycerol-3-phosphate/glycerol-2-phosphate $A B C$ transporter complex. As shown in Fig. 1c, module 76 and module 112 were perfectly matched with the $Y h d W /$ $Y h d X / Y h d Y / Y h d Z \mathrm{ABC}$ transporter complex and the galactofuranose/galactopyranose $\mathrm{ABC}$ transporter complex, respectively. The analysis above indicated that ELPA is an effective method to predict E. coli protein complexes in PPI network.

\section{Prediction of the functional module}

Those protein modules of net $\mathrm{H}$ and netC identified by ELPA were matched with benchmark functional annotations of $E$. coli in the EcoCyc dataset, respectively. If the Matching Score between a protein module and a given GO term was greater than 0.5 , this protein module was recognized as a potential functional module. In netH, most prediction modules $(82.5 \%)$ were significant functional modules, of which about 30\% exactly matched a certain functional term (Matching Score equal to 1). For example, as shown in Fig. 2a, module 19 contained 25 proteins, of which 24 proteins matched GO: 0006810. Obviously, it was a functional module. All the seven proteins of module 40 were completely covered by GO: 0005886, GO:0016020, and GO:0017004, respectively (as shown in Fig. 2b). In netC, $91.6 \%$ of predicted modules were significant functional modules, of which $53.2 \%$ were completely covered by at least one GO term. For example, as shown in Fig. 2c and d, module 9 contained seven proteins: $m f d$, nus $A$, pyrG, rpoB, rpsE, rps $U$, and $u v r A$, which were completly annotated by GO:0005829. Similarly, ten proteins of module 26: $d p p A, d p p B, d p p C$, $d p p D, d p p F, n i k A, o p p A, o p p B, o p p D$, and $\operatorname{sap} A$ were all annotated with GO:0006810. The analysis above indicated that ELPA is an effective method to predict E. coli functional modules in PPI network.

\section{Comparative analysis of predicted protein complexes and functional modules}

A protein complex is a physical aggregation of several proteins that interact at the same time and location through molecular interactions. A functional module also consists of multiple proteins that interact with each other to control or perform a particular cellular function. However, unlike protein complexes, these proteins do not necessarily interact at the same time and location. Therefore, the comparative analysis of protein complexes and corresponding functional modules is of great scientific significance.

For example, as shown in Fig. 3a, module 21 in NetH was mainly composed of three real complexes: NADH: ubiquinone oxidoreductase I, hydrogenase 4 and formate hydrogenlyase. All proteins in the hydrogenase 4

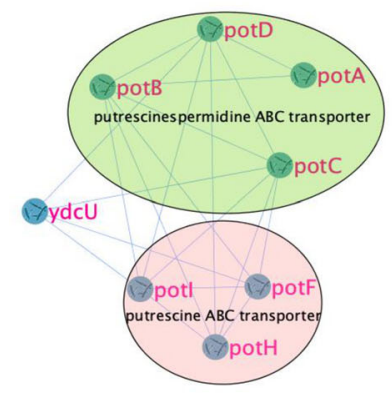

(a)

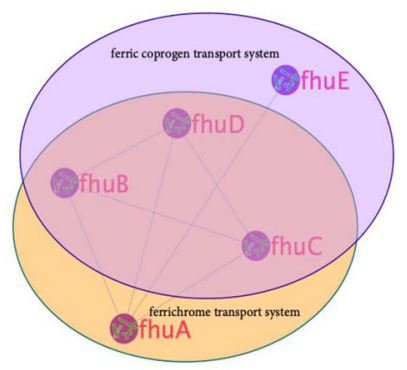

(b)

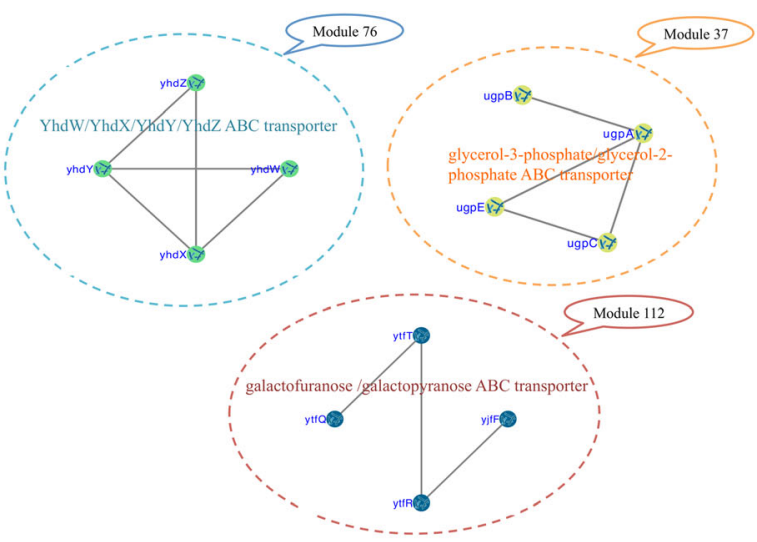

(c)

Fig. 1 Predicted protein complexes in netH. a The proteins of module 50 in netH matched with real complexes. $\mathbf{b}$ The proteins of module 54 in netH matched with real complexes. c Three predicted modules perfectly matched with the corresponding protein complexes in netC 


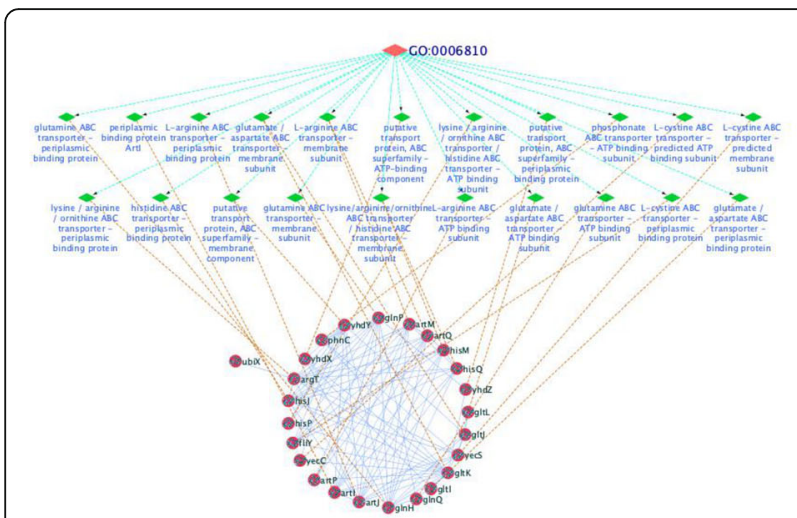

(a)

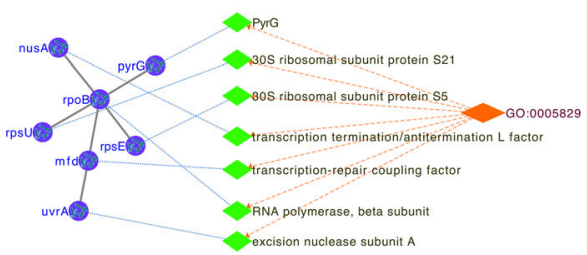

(c)

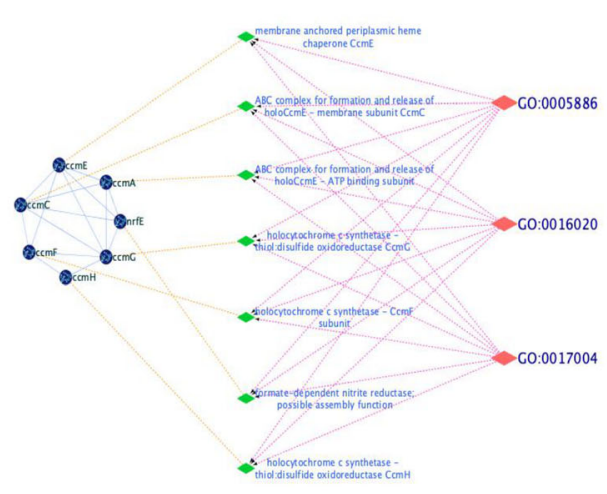

(b)

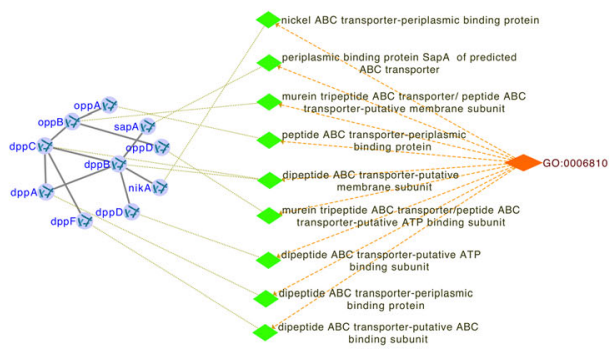

(d)

Fig. 2 Predicted functional modules in netH and netC. a The proteins of module 19 in netH matched with GO annotations. b The proteins of module 40 in netH matched with GO annotations. c The proteins of module 9 in netC matched with $\mathrm{GO}$ annotations. $\mathbf{d}$ The proteins of module 26 in netC matched with GO annotations

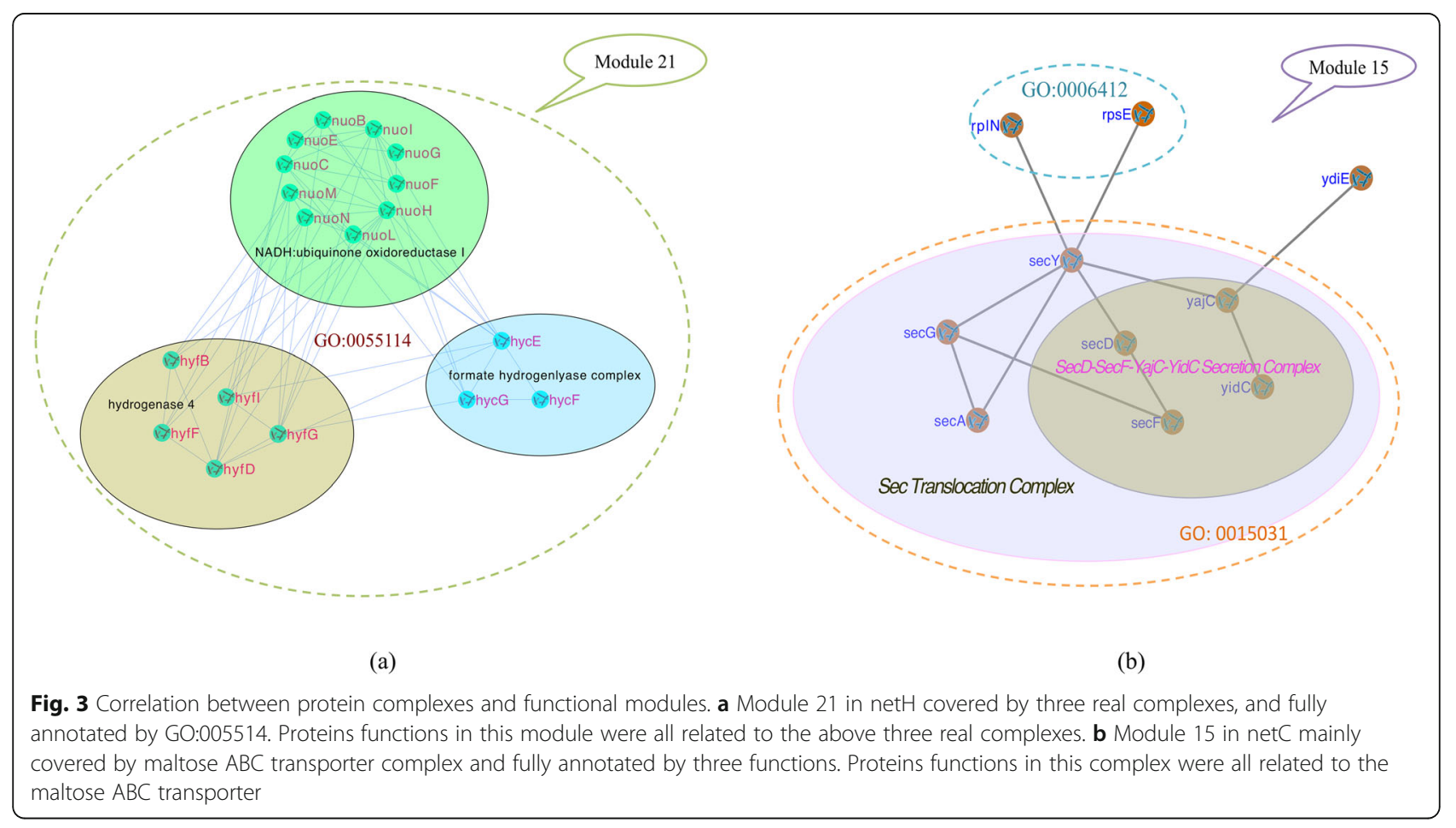


complex were completely covered by this module, 10 out of 11 proteins of ubiquinone oxidoreductase I complex matched this module, and 3 out of 5 proteins of formate hydrogenlyase complex matched this module. Also, we noticed that GO: 0055114 completely covered the 18 proteins of this module, which meant that the three protein complexes mentioned above might combine to perform a certain function. $h y c E$, $h y c F$ and $h y c G$ were the components of the formate hydrogenlyase complex, and their functional annotations were hydrogenase 3 , formate hydrogenlyase complex iron-sulfur protein, and hydrogenase 3 and formate hydrogenlyase complex-HycG subunit, respectively. This meant that the functions of these three proteins were consistent with the formate hydrogenlyase complex, and it also implied that the hydrogenase 3 complex was related to the formate hydrogenlyase complex. The Hydrogenase 4 complex proteins: hyfB, hyfD, hyfF, hyfG and hyfI were annotated with hydrogenase 4-component B, D, F, and large, small subunit respectively. This indicated that the functions of these five proteins and the hydrogenase 4 complex were highly coherent. The ten remaining proteins of this module: nиов, пиоC, nиоE, nиоF, nиоG,

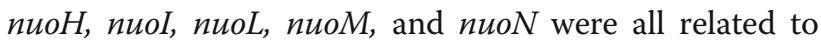
NADH: ubiquinone oxidoreductase complex. Therefore, we predicted that the above three protein complexes might significant functional correlations.

Figure $3 \mathrm{~b}$ showed another similar case. Module 15 in netC consisted of ten proteins and contained two real complexes: Sec Translocation Complex and SecD-SecFYajC-YidC Secretion Complex. All four proteins of SecD-SecF-YajC-YidC Secretion Complex were covered by this module and Sec Translocation Complex, and they were all matched with GO:0016021, among them yidC were annotated with inner-membrane protein insertion factor. 7 out 9 proteins of Sec Translocation Complex matched this module, and the seven proteins: $\sec A$, secD, $\sec F, \sec G, \sec Y$, yajC and yidC were enriched with the term "protein transport" of GO: 0015031, which meant their functions were consistent with the complex. Besides one uncharacterized protein $y d i E$, the two remaining proteins $r p l N$ and $r p s E$ were enriched with the term "translation" of GO: 0006412, and the term "rRNA binding" of GO:0019843, respectively. The results above indicated that those predicted protein modules are significant related to corresponding protein complexes and functional modules. Usually, a functional module might cover more than one protein complex.

\section{Comparison with MCL}

Most biological network module identification methods were based on node clustering, and among them, MCL has been proven to be superior to other methods in identifying the protein modules in most cases [23, 26, 27]. ELPA is a module identification method based on link clustering. It considered the attitudes of nodes and links at the same time, and can better reflect the network structure than nodes [21, 28]. ELPA is a parameter-free method, and MCL used the default parameters. And the clustering results of ELPA and MCL were compared in both networks, respectively.

Three metrics: Precision, Recall, F-measure were utilized to compare the performance of MCL and ELPA in predicting protein complexes. In netH, the performance comparison of two methods for predicting protein complexes was shown in Fig. 4a. The values of Precision, Recall and F-measure of ELPA were $72.5,61.5$, and $66.5 \%$, respectively, while the corresponding results of MCL were 55.1, 65.5 , and $59.9 \%$, respectively. Similar results were obtained in netC. As shown in Fig. 4c, the values of Precision, Recall and F-measure of ELPA were 67, 62.3 and 64.6\%, while the corresponding results of MCL were 67.9, 60 and $63.7 \%$, respectively. From the Fig. 4, we found that the performance of ELPA and MCL is comparable in the prediction of protein complexes. For example, in netH, Flagellum complex matched with module 10 of ELPA and module 59 of $\mathrm{MCL}$, and the corresponding matching scores were 58.9 and $43 \%$, respectively. Enterobactin synthase complex matched with module 53 of ELPA and module 46 of MCL, and corresponding matching scores were 50 and 45\%, respectively. SecD-SecF-YajC-YidC Secretion complex matched with module 69 of ELPA and module 149 of MCL, and the corresponding matching scores were 56.3 and $45 \%$, respectively.

In general, Precision and AMS are two effective metrics to evaluate the predicted quality of functional modules. The performance comparison of two methods for predicting functional modules was shown in Fig. 4b. Values of Precision and AMS of ELPA were 82.5 and $70.9 \%$, respectively, and that of MCL were 85.9 and $70 \%$, respectively. Analogous results were obtained in netC. As shown in Fig. 4d, values of Precision and AMS of ELPA were 91.6 and $77.5 \%$, while the corresponding results of MCL were 93 and $77.8 \%$, respectively. We concluded that the performance of both methods is comparable in the prediction of functional modules. For example, in netC, both module 190 of ELPA and module 66 of MCL were enriched in the GO: 0000018, and corresponding matching scores were 100 and $66.7 \%$, respectively. Both module 29 of ELPA and module 101 of MCL were enriched in the GO: 0006281, and corresponding matching scores were 100 and $75 \%$, respectively. Both module 83 of ELPA and module 168 of MCL were enriched in the GO: 0009060, and the corresponding matching scores were 80 and $75 \%$, respectively. The results above showed that ELPA is an effective method to predict $E$. coli protein complexes and functional modules. 


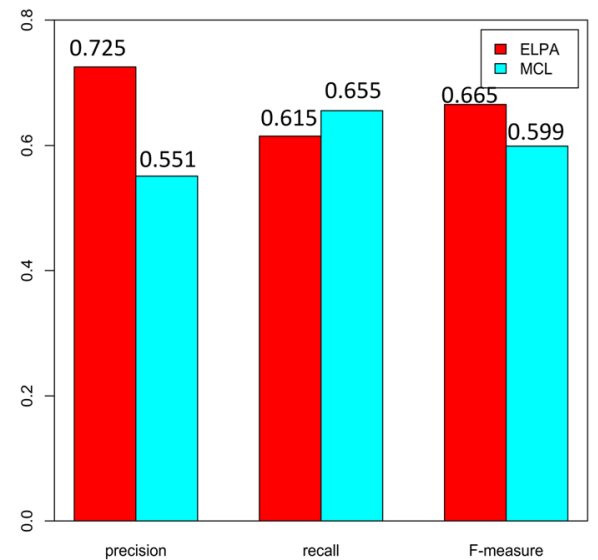

(a)

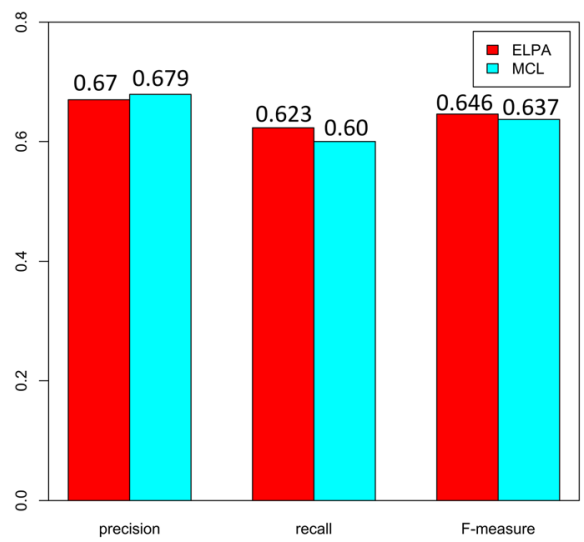

(c)

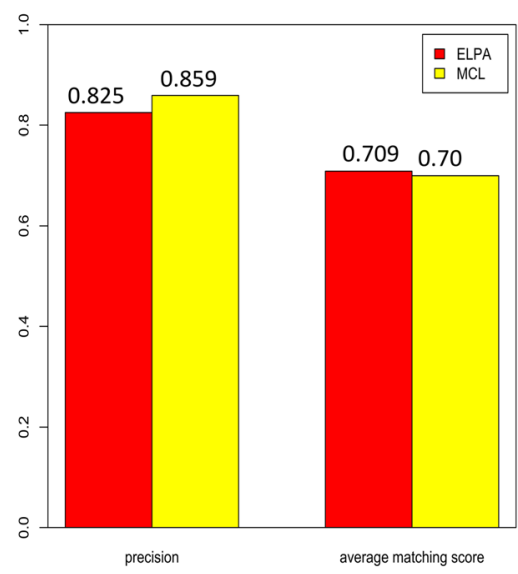

(b)

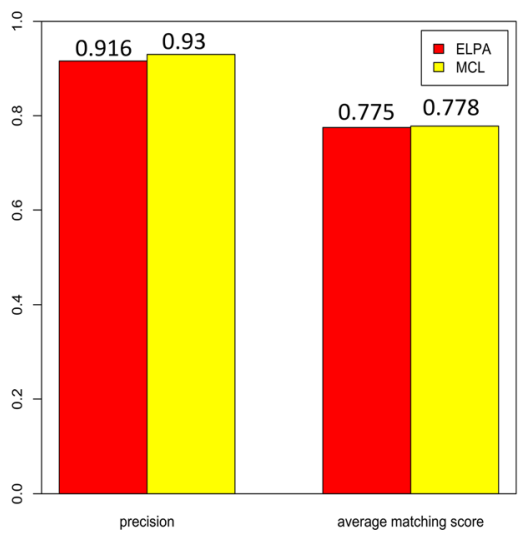

(d)

Fig. 4 Comparative performance of ELPA and MCL in netH and netC. a Comparison of protein complex predictions in netH. b Comparison of functional modules predictions in netH. c Comparison of protein complex predictions in netC. $\mathbf{d}$ Comparison of functional modules predictions in netC

\section{Discussion}

Besides well-characterized protein complexes and functional modules, we also identified modules that had not been matched to an EcoCyc protein complex or functional category in both networks. In netH and netC, we discovered 36 and 67 novel protein complexes, and 21 and 17 novel functional modules, respectively (Table S2). A notable example of a novel protein complex was module 36 in netH, composed of five proteins: pheS, pheM, thrS, argS and erfK. ThrS is also a translational repressor protein, and it controls binds its own mRNA in the operator region upstream of the start codon. ThrRS represses translation by preventing the ribosome from to mRNA, and tRNA acts as an antirepressor allowing fine level control of enzyme synthesis. ThrS, argS, pheS and pheM are all involved in aminoacyl-tRNA ligase activity, and they are the members of arginine-tRNA ligase, phenylalanine-tRNA ligase alpha chain, phenylalanyltRNA synthetase operon leader peptide and threoninetRNA ligase superfamily, respectively. In addition, pheS,
thrS and $\arg S$ are in Aminoacyl-tRNA biosynthesis pathway, and pheM is significant related to Aminoacyl-tRNA biosynthesis. We knew little about erfK except that it was involved in the peptidoglycan biosynthesis pathway. These annotations indicated that these proteins might constitute a protein complex involved in aminoacyltRNA biosynthesis.

An example for the novel functional module was module 10 in netC, and it consisted of seven proteins: $\operatorname{rps} N$, rpmC, rplV, rlmJ, sbcB, holC and hflC. RpsN binds 16S rRNA, required for the assembly of $30 \mathrm{~S}$ particles and may also be responsible for determining the conformation of the 16S rRNA at the A site. RplV binds specifically to $23 \mathrm{~S}$ rRNA. Its binding is stimulated by other ribosomal proteins, and makes multiple contacts with different domains of the $23 \mathrm{~S}$ rRNA in the assembled $50 \mathrm{~S}$ subunit and ribosome. RpmC binds $23 \mathrm{~S}$ rRNA, and contacts trigger factor. RlmJ specifically methylates the adenine in position 2030 of $23 \mathrm{~S}$ rRNA, and it required for the utilization of extracellular DNA as the sole source of 
carbon and energy. $\mathrm{HolC}$ is part of the beta sliding clamp loading complex, which hydrolyzes ATP to load the beta clamp onto primed DNA to form the DNA replication pre-initiation complex. $S b c B$ degrades singlestranded DNA (ssDNA) in a highly possessive manner, and also functions as a DNA deoxyribophosphodiesterase that releases deoxyribose-phosphate moieties following the cleavage of DNA at an apurinic/apyrimidinic (AP) site by either an AP endonuclease or AP lyase. $H f l C$ controls the lysogenization frequency of phage lambda. Together, these annotations suggested that these proteins form part of a translation module.

\section{Conclusion}

In this paper, a link clustering algorithm (ELPA) was used to identify protein complexes and functional modules in the E. coli PPI network. Through comparison with the EcoCyc database, we have discovered some novel and interesting complexes and functional modules. In addition, we compared and analyzed protein complexes and functional modules derived from the same predicted protein modules. It was found that protein complexes are highly overlapped with the corresponding functional modules, many of which contain more than one protein complex, which helps to understand the dynamic relationship between protein complexes and functional modules. Finally, the results of ELPA were compared with that of MCL, and we found that their performance is comparable in most cases. Therefore, we concluded that ELPA can be used as an effective cluster analysis tool for different types of biological networks. In further work, we will explore the key regulatory proteins and pathways in the transcriptional regulatory network of $E$. coli, based on the corresponding protein complexes and functional modules.

\section{Methods}

\section{Source of PPI data}

Two high-throughput experiments of protein-protein interactions (PPIs) datasets of E. coli were retrieved from the original paper of $\mathrm{Hu}$ et al. [5] and Cong et al. [8], respectively. Most of the data from these resources came from Yeast-Two- Hybrid $(\mathrm{Y} 2 \mathrm{H})$ and Tandem Affinity Purification (TAP). The dataset provided by $\mathrm{Hu}$ et al. included a large-scale TAP-derived network and a functional network. Our analysis merged the two networks into a single combined network which contained 7613 interactions among 2283 proteins. The dataset recently published by Cong et al. is a large-scale $\mathrm{Y} 2 \mathrm{H}$-derived network of $E$. coli, which contained 1618 interactions among1, 476 proteins. To predict protein complexes more efficiently, only those binary interactions associated with known complex proteins were considered. As a result, 3280 interactions among 1298 proteins were retrieved from the dataset of $\mathrm{Hu}$ et al., and 1299 interactions among 785 proteins were retrieved from the dataset of Cong et al.

\section{Benchmark for protein complexes and functional annotations}

In most studies, protein complexes and functional annotations of E. coli downloaded from EcoCyc database [29] were regarded as the "gold standard", and all these data sets downloaded are up-to-date. As we all know, many protein complexes of E. coli contain only two proteins, thus those complexes containing at least two proteins in the E. coli K-12 dataset of EcoCyc were kept as benchmark complexes. In this way, we obtained 295 benchmark protein complexes containing 732 proteins. Furthermore, the EcoCyc Gene Ontology (GO) database was taken as the benchmark functional classes of $E$. coli. In total, we obtained three protein datasets: $\mathrm{EcoCyc}, \mathrm{Hu}$ et al., and Cong et al., and we found the relationships among them by Venn diagrams (shown in Fig. 5).

\section{Prediction of protein modules}

In the past decade, many clustering algorithms for complex networks have been developed, most of which are based on node clustering. However, only a few methods can be used in complex biological networks. In this paper, an algorithm based on link clustering: Edge Label Propagation Algorithm (ELPA) [21], was utilized to identify the protein modules of $E$. coli in the above two PPI networks. The original paper [21] have shown that the performance of ELPA outperforms other link clustering algorithms [30,31]. In contrast to node clustering, link clustering has the natural advantages of being compatible with the node attributes and link attributes of complex networks and can reflect the network topology structure better. Then, the protein modules detected by ELPA were matched with the "gold standard" protein complexes and functional annotations of EcoCyc and predicted the meaningful protein complex modules and protein functional modules.

\section{Evaluation methods}

Two criteria were employed to evaluate the performance of ELPA. One was matching the identified protein modules with known protein complex of EcoCyc benchmark dataset. The other was the functional enrichment of the identified protein modules.

To determine the matching efficiency of a predicted complex $p$ and corresponding real complex $b$ in the benchmark complex set, the Matching Score $\left(M S_{p b}\right)$ between them was calculated as [32]: 


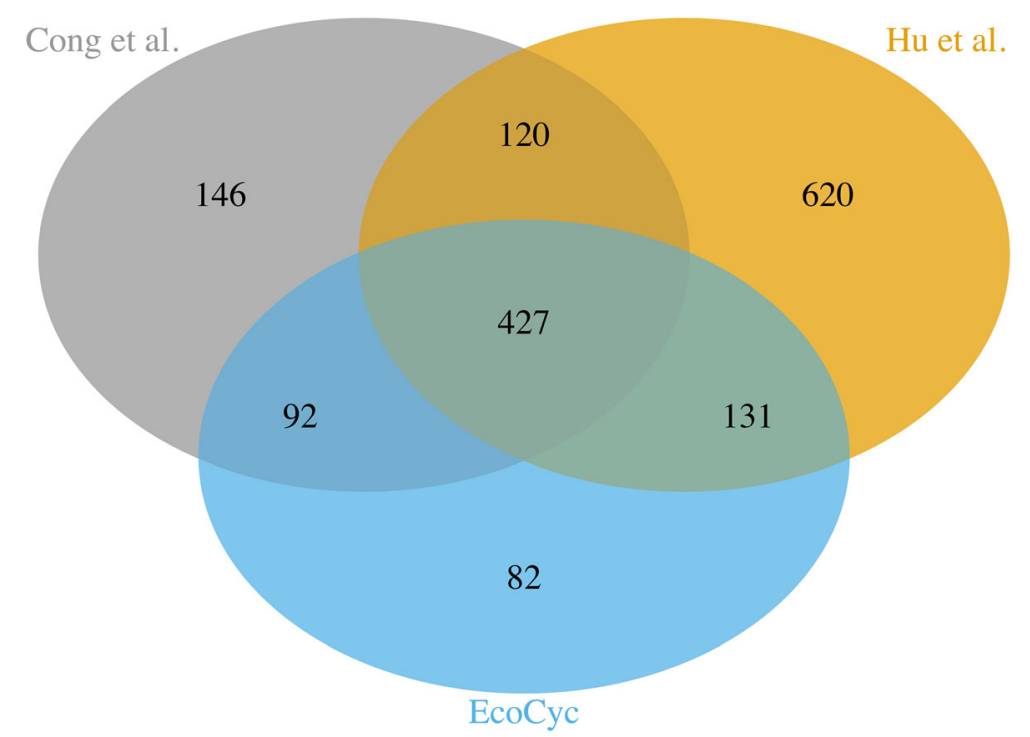

Fig. 5 The relationships among three protein datasets (EcoCyc, Hu et al. and Cong et al.)

$$
M S_{p b}=\frac{n_{p b}^{2}}{n_{p} \cdot n_{b}}
$$

Where $n_{p b}$ is the number of proteins shared by the predicted complex $p$ and the real complex $b, n_{p}$ is the number of proteins in complex $p$, and $n_{b}$ is the number of proteins in complex $b$. A predicted complex and a real complex were considered to be a match if their matching score was no less than a specific threshold (typical threshold is $0.2[24,26]$ ).

To evaluate the predicted protein complexes, we checked how well the predicted complexes matched the actual complexes. Three types of popular evaluation criteria: Precision, Recall and F-measure [28], were used to quantify the quality of the predicted protein complexes. Let $P$ and $B$ denote the set of predicted and actual complexes, respectively. Let $N_{P}$ denote the number of protein complexes, and let $N_{B}$ denote the number of actual complexes in the benchmark dataset. Let $N_{P C}$ denote the number of predicted complexes that matched at least one real complex, and let $N_{B C}$ be the number of actual complexes that matched at least one predicted complex. Precision and Recall were then defined as follows:

$$
\begin{aligned}
& \text { Precision }=\frac{N_{P C}}{N_{P}} \\
& \text { Recall }=\frac{N_{B C}}{N_{B}}
\end{aligned}
$$

$$
F=2 \times \frac{\text { Precision } \times \text { Recall }}{(\text { Precision }+ \text { Recall })}
$$

The arithmetic mean of matching score (AMS) was another metric to evaluate the predicted protein complexes.

To evaluate the functional enrichment of a predicted protein module, the matching score between a predicted protein module and a given GO term was used to estimate whether the proteins in the predicted module were enriched for the GO term. Then, Precision and AMS were used to evaluate predicted functional modules.

\section{Supplementary information}

Supplementary information accompanies this paper at https://doi.org/10. 1186/s12866-020-01904-6.

Additional file 1. S1-clustering results

Additional file 2. S2-novel complexes and functional modules

\section{Abbreviations}

E. coli: Escherichia coli; PPI: Protein-protein interaction; ELPA: Edge label propagation algorithm; MCL: Markov clustering algorithm; Y2H: Yeast-TwoHybrid; TAP: Tandem Affinity Purification; AMS: Arithmetic mean of Matching Score

\section{Acknowledgments}

We thank those researchers who have graciously shared the E. coli PPls with us.

\section{Authors' contributions}

Methodology, validation, formal analysis and writing_original draft preparation, W.L.; writing, review, visualization and editing, P.K.\& G.H. All the authors have read and approved the manuscript. 


\section{Funding}

This research was funded by Talent Foundation of Ludong University (LA2016007), Natural Science Foundation of Jiangsu (BK2016245), Natural Science Foundation of Shandong (ZR2017MF052), National Natural Science Foundation of China (81830052), Construction project of Shanghai Key Laboratory of Molecular Imaging (18DZ2260400), Shanghai Municipal Education Commission (Class II Plateau Disciplinary Construction Program of Medical Technology of SUMHS, 2018-2020), and "Climbing" program from SUMHS (B3-0200-20-311007). These funding bodies did not participate in study design, data collection, analysis and interpretation or writing of the manuscript.

\section{Availability of data and materials}

All datasets used in this paper are public and public access to all databases is open. The data of Cong et al. is available at https://science.sciencemag. org/content/365/6449/185/tab-figures-data, the data of Hu et al. is available at http://compsysbio.org/bacteriome/download.php, and EcoCyc E. coli protein complexes and functional annotations dataset is available at https:// ecocyc.org/.

\section{Ethics approval and consent to participate}

Not applicable.

\section{Consent for publication}

Not applicable.

\section{Competing interests}

The authors declare that they have no competing interests.

\section{Author details}

'Shanghai Key Laboratory of Molecular Imaging, Shanghai University of Medicine and Health Sciences, Shanghai 201318, China. ${ }^{2}$ Research Center for Intelligence Information Technology, Nantong University, Nantong 226019, Jiangsu, China. ${ }^{3}$ School of Mathematics and Statistics Science, Lu Dong University, Yantai 264025, Shandong, China.

\section{Received: 11 March 2020 Accepted: 14 July 2020}

Published online: 06 August 2020

\section{References}

1. Arifuzzaman M, Maeda M, Itoh A, et al. Largescale identification of proteinprotein interaction of Escherichia coli K-12. Genome Res. 2006;16:686-91.

2. Butland G, Peregrin-Alvarez JM, Li J, et al. Interaction network containing conserved and essential protein complexes in Escherichia coli. Nature. 2005; 433(7025):531-7.

3. Joyce AR, Reed JL, White A, et al. Experimental and computational assessment of conditionally essential genes in Escherichia coli. J Bacteriol. 2006;188:8259-71.

4. Riley M, Abe T, Arnaud MB, et al. Escherichia coli K-12: a cooperatively developed annotation snapshot-2005. Nucleic Acids Res. 2006;34:1-9.

5. Hu P, Janga SC, Babu M, et al. Global functional atlas of Escherichia coli encompassing previously uncharacterized proteins. PLoS Biol. 2009;7(4):929-47.

6. Butland $G$, Joyce $L$, Wehong $Y$, et al. Interaction network containing conserved and essential protein complex in Escherichia coli. Nature. 2005; 433:531-7.

7. Seesandra V. Rajagopala et al. the binary protein-protein interaction landscape of Echerichia coli. Nat. Biotechnol. 2014;32:285-90.

8. Cong Q, Anishchenko I, Ovchinnikov S, Baker D. Protein interaction networks revealed by proteome coevolution. Science. 2019;365(6449):185-9. https://doi.org/10.1126/science.aaw6718

9. Babu M, Bundalovic-Torma C, Calmettes C, et al. Global landscape of cell envelope protein complexes in Escherichia coli. Nat Biotechnol. 2018:36: 103-12.

10. Spirin V, Mirny LA. Protein complexes and functional modules in molecular networks. Proc Natl Acad Sci U S A. 2003;100(21):12123-8.

11. Shi L, Lei X, Zhang A. Protein complex detection with semi-supervised learning in protein interaction networks. Proteome Sci. 2011:9 supppl 1:S5.

12. Osbaldo RA, Julio A, Ricardo MM, et al. Modular analysis of the transcriptional regulatory network of E. coli. TRENDS in Gentetics. 2005;21(1): $16-20$.
13. Faith JJ, Hayete B, Thaden JT, et al. Largescale mapping and validation of Escherichia coli transcriptional regulation from a compendium of expression profiles. PLoS Biol. 2007;5:e8.

14. Barrett $\mathrm{CL}$, Herring $\mathrm{CD}$, Reed $\mathrm{JL}$, Palsson $\mathrm{BO}$. The global transcriptional regulatory network for metabolism in Escherichia coli exhibits few dominant functional states. Proc Natl Acad Sci U S A. 2005;102:19103-8.

15. Gama-Castro S, Jimenez-Jacinto V, Peralta-Gil M, et al. RegulonDB (version 6. $0)$ : gene regulation model of Escherichia coli K-12 beyond transcription, active (experimental) annotated promoters and Textpresso navigation. Nucleic Acids Res. 2008:36:D120-4.

16. Fang X, Sastry A, Mih N, et al. Global transcriptional regulatory network for $\backslash \mathrm{r}$, Escherichia colivr, robustly connects gene expression to transcription factor activities. Proc Natl Acad Sci. 2017;114(38):10286-91.

17. Lempp M, Farke N, Kuntz M, et al. Systematic identification of metabolites controlling gene expression in E coli. Nat Commun. 2019;10:4463.

18. Feist AM, Henry CS, Reed JL, et al. A genome-scale metabolic reconstruction for Escherichia coli K-12 MG1655 that accounts for 1260 ORFs and thermodynamic information. Mol Syst Biol. 2007;3:121.

19. Geryk J, Sianina F. Modules in the metabolic network of E. coli with regulatory interactions. Int J Data Min Bioinform. 2013;8(2):188-202.

20. Peregrin-Alvarez JM, Xiong X, Su C, et al. The modular organization of protein interactions in Echerichia coli. PLoS Comput Biol. 2009;5(10): e1000523.

21. Liu W, Jiang X, Pellegrini $M$, et al. Discovering communities in complex networks by edge label propagation. Sci Rep. 2016;6:22470.

22. Enright AJ, Van Dongen S, Ouzounis CA. An efficient algorithm for largescale detection of protein families. Nucleic Acids Res. 2002;30:1575-84.

23. Li X, Wu M, Kwoh CK, et al. Computational approaches for detecting protein complexes from protein interaction networks: a survey. BMC Genomics. 2010;11Suppl 1:1-19.

24. Ji J, Zhang A, Liu C, et al. Survey: functional module detection from proteinprotein interaction networks. IEEE Trans Knowl Data Eng. 2014;26(2):261-77.

25. Yumeng $L$, Xiaolong $W$, Bin $L$. A comprehensive review and comparison of existing computational methods for intrinsically disordered protein and region prediction. Brief Bioinform. 2019;20(1):330-46.

26. Brohee $\mathrm{S}$, van Helden J. Evaluation of clustering algorithms for proteinprotein interaction networks. BMC Bioinformatics. 2006:7:488.

27. Reid AJ, Ranea JA, Orengo CA. Comparative evolutionary analysis of protein complexes in E. coli and yeast. BMC Genomics. 2010;11:79-0.

28. Liu W, Wu A. Uncover protein complexes in E coli network. In: IEEE international conference on bioinformatics and biomedicine; 2015. https:// doi.org/10.1109/BIBM.2015.7359844.

29. Keseler IM, Mackie A, Peraltagil M, et al. EcoCyc: fusing model organism databases with systems biology. Nucleic Acids Res. 2013;41:D605-12.

30. Ahn YY, Bagrow JP, Lehmann S. Link communities reveal multi-scale complexity in networks. Nature. 2010:466:761-4.

31. He D, Liu D, Zhang W, Jin D, Yang B. Discovering link communities in complex networks by exploiting link dynamics. J Stat Mech. 2012;10:P10015.

32. Chen B, Fan W, Liu J, et al. Identifying protein complexes and functional modules-from static PPI networks to dynamic PPI networks. Brief Bioinform. 2014;15:177-94.

\section{Publisher's Note}

Springer Nature remains neutral with regard to jurisdictional claims in published maps and institutional affiliations.

Ready to submit your research? Choose BMC and benefit from:

- fast, convenient online submission

- thorough peer review by experienced researchers in your field

- rapid publication on acceptance

- support for research data, including large and complex data types

- gold Open Access which fosters wider collaboration and increased citations

- maximum visibility for your research: over $100 \mathrm{M}$ website views per year

At $\mathrm{BMC}$, research is always in progress.

Learn more biomedcentral.com/submissions 